\title{
A Metabolic Race
}

\author{
Uma Corrida Metabólica
}

\author{
Amanda Costa', Juliana Apolinário², Félix Barros ${ }^{3}$, André Santos ${ }^{4}$, Cátia Sodré 1 \\ ${ }^{1}$ Departamento de Biologia Celular e Molecular, Instituto de Biologia, Universidade Federal Fluminense (UFF) - \\ Niterói - RJ-Brasil \\ ${ }^{2}$ Arquitetura UNIAN - RJ - Brasil \\ ${ }^{3}$ Curso de Automação Industrial, CEFET-RJ - Campus Maria da Graça - RJ - Brasil \\ ${ }^{4}$ Departamento de Microbiologia Geral, Instituto de Microbiologia Paulo de Goés, UFRJ - RJ - Brasil \\ *e-mail: amandamendonca@id.uff.br
}

\begin{abstract}
It is common among medical students and other courses in biomedical area, to raise questions about the applicability of certain biochemical concepts. Since such contents will be present throughout their professional lifes, it is essential to emphasize the relevance of the basic cycle, stage of the medical training in which biochemistry teaching is inserted. With the aim of bring together theory and future clinical practice, a board game was developed based on a biochemical approach of Metabolic Syndrome (MS) clinical cases. The game reproduces the environment and routine experienced by students of Federal Fluminense University, in which players are divided into "patients and doctors" and move on a simplified map of Niterói city. Questions and challenge cards are used to test students' biochemical knowledge and a scoring system based on three of the five criteria to diagnose MS, is used to provide proportional diagnoses to the each player's performance.
\end{abstract}

Keywords: game; metabolism; teaching.

\section{Resumo}

Dentre os estudantes de medicina e de outros cursos da área biomédica, é comum surgirem questionamentos sobre a aplicabilidade de determinados conceitos bioquímicos. Uma vez que tais conteúdos estarão presentes ao longo de toda a vida profissional, enfatizar a relevância do ciclo básico, etapa da formação médica na qual está inserido o ensino da bioquímica, é imprescindível. Com o objetivo de aproximar a teoria da futura prática clínica, foi desenvolvido um jogo de tabuleiro baseado na abordagem bioquímica de casos clínicos sobre Síndrome Metabólica (SM). O jogo reproduz o ambiente e a rotina vivenciada pelos estudantes da Universidade Federal Fluminense, no qual os jogadores dividem-se em pacientes e médicos e movimentam-se em um mapa simplificado da cidade de Niterói. São utilizadas fichas de perguntas e desafios para testar os conhecimentos bioquímicos dos alunos e um sistema de pontuação baseado em três dos cinco critérios de diagnóstico da SM, fornecendo diagnóstico proporcional ao desempenho de cada jogador.

Palavras-chave: jogo; metabolismo; ensino. 
Ficha da atividade

\begin{tabular}{|c|c|}
\hline Título & Uma Corrida Metabólica \\
\hline Categoria: & $\begin{array}{l}\text { Prêmio Nacional de Ensino de Bioquímica e Biologia Molecular "Bayardo } \\
\text { Baptista Torres": Materiais didáticos }\end{array}$ \\
\hline $\begin{array}{l}\text { Tipo de } \\
\text { Material }\end{array}$ & Jogo - Construção de Jogo \\
\hline Público-alvo: & Estudantes que estejam cursando ou já tenham cursado a disciplina Bioquímica. \\
\hline $\begin{array}{l}\text { Conteúdos } \\
\text { abordados }\end{array}$ & $\begin{array}{l}\text { Abordagem bioquímica da Síndrome Metabólica e Diabetes Mellitus, conteúdos } \\
\text { de bioquímica metabólica (glicólise, ciclo de Krebs, cadeia respiratória, } \\
\text { metabolismo do glicogênio, metabolismo dos lipídios, metabolismo dos } \\
\text { aminoácidos, gliconeogênese, formação de corpos cetônicos, metabolismo do } \\
\text { colesterol). }\end{array}$ \\
\hline $\begin{array}{l}\text { Objetivos } \\
\text { educacionais }\end{array}$ & $\begin{array}{l}\text { Revisar e consolidar, de forma lúdica e dinâmica, conteúdos trabalhados na } \\
\text { apresentação e discussão de casos clínicos sobre Síndrome Metabólica e nas } \\
\text { demais aulas de bioquímica metabólica. Tais objetivos serão alcançados através } \\
\text { das fichas de perguntas e desafios que testam os conhecimentos bioquímicos } \\
\text { dos jogadores e por meio dos comandos presentes no tabuleiro, que provocam } \\
\text { alterações nos valores de Glicemia, Circunferência Abdominal e HDL (High } \\
\text { Density Lipoprotein). A escolha desses três parâmetros deve-se a sua } \\
\text { importância para o diagnóstico laboratorial da Síndrome Metabólica. Vale } \\
\text { ressaltar que, a realização desta atividade em grupo promove uma maior } \\
\text { interação entre os alunos. }\end{array}$ \\
\hline $\begin{array}{l}\text { Duração } \\
\text { estimada }\end{array}$ & Aproximadamente de 30 a 40 minutos. \\
\hline $\begin{array}{l}\text { Materiais } \\
\text { utilizados }\end{array}$ & $\begin{array}{l}\text { Um tabuleiro com oito pinos, um dado com números de } 1 \text { a } 6 \text {, fichas de } \\
\text { perguntas (64), fichas desafio (48), uma ficha de diagnósticos e um bloco para } \\
\text { anotações do "médico". }\end{array}$ \\
\hline Justificativa & $\begin{array}{l}\text { A Síndrome Metabólica descreve um conjunto de sinais e sintomas metabólicos } \\
\text { que aumenta, significativamente, as chances de um indivíduo desenvolver } \\
\text { resistência à insulina e doenças cardiovasculares. Alguns aspectos contribuem } \\
\text { para o seu desenvolvimento, como: a genética, o sobrepeso e o sedentarismo. } \\
\text { Dada a relevância desse tema e a sua crescente incidência, principalmente, } \\
\text { entre os americanos, foi introduzida no conteúdo programático da disciplina de } \\
\text { Bioquímica a apresentação e discussão de casos clínicos sobre este tema, } \\
\text { ressaltando a importância clínica de fenômenos bioquímicos envolvidos na } \\
\text { fisiopatologia da síndrome. Dentro desse contexto, o jogo de tabuleiro funciona } \\
\text { como ferramenta educativa que auxilia o entendimento e a discussão dos casos } \\
\text { clínicos, além de permitir a abordagem de diversos conteúdos previamente } \\
\text { trabalhados, consolidando-os de forma dinâmica e divertida. Além disso, é } \\
\text { também uma estratégia de conscientização e reflexão de modo de vida, já que } \\
\text { demonstra quão próxima a Síndrome Metabólica está do dia-a-dia dos } \\
\text { estudantes. }\end{array}$ \\
\hline
\end{tabular}




\section{Introdução}

A utilização de jogos didáticos como estratégia para educação na área da saúde tem sido considerada, por muitos autores [1-6], um instrumento educativo capaz de contribuir para a construção e consolidação de conhecimentos relevantes na área biomédica [6].

A abordagem de forma lúdica e estimulante mostra-se eficaz na participação do estudante no entendimento e reforço dos conteúdos abordados em sala de aula. Quando estudantes de graduação em medicina, por exemplo, cursam as disciplinas do ciclo básico, por vezes, se perguntam qual a aplicabilidade de alguns conceitos bioquímicos que são abordados e discutidos em sala de aula. É inquestionável, no entanto, a importância desta primeira etapa na formação médica, pois tais conteúdos estarão presentes durante toda a vida profissional do médico.

Partindo desse pressuposto, a inserção de casos clínicos no conteúdo programático da disciplina de Bioquímica já nos primeiros períodos do curso de Medicina, apontam a importância de tais conhecimentos "básicos" para um futuro entendimento clínico. Tal proposta se consolida cada vez mais como instrumento positivo e integrador a outras disciplinas do ciclo básico e clínico do curso. Dentro desse contexto, o objetivo deste projeto foi desenvolver um jogo de tabuleiro que se apresenta como mais uma ferramenta educativa, cujo objetivo principal é consolidar, a partir de uma abordagem bioquímica, alguns conceitos discutidos durante a apresentação de casos clínicos sobre Síndrome Metabólica, assim como rever conteúdos previamente trabalhados na disciplina.

Tais objetivos são alcançados através dos comandos presentes no tabuleiro, que provocam alterações nos valores de Glicemia, Circunferência Abdominal e HDL (High Density Lipoprotein). Vale ressaltar que a escolha destes três parâmetros deve-se a sua importância para o diagnóstico laboratorial da Síndrome Metabólica, segundo critérios brasileiros [7].

$\mathrm{O}$ jogo foi organizado de forma a mimetizar o ambiente e a rotina vivenciada pelos estudantes da Universidade Federal Fluminense (UFF), que "percorrem" durante o jogo um mapa simplificado da cidade de Niterói, no qual são destacados alguns locais, como o Campus do Valonguinho, o Hospital Universitário Antônio Pedro (HUAP) e a Praia de Icaraí.

Além desses aspectos familiares, os comandos presentes no tabuleiro foram planejados visando uma identificação, por parte dos estudantes, quanto aos seus hábitos de vida (alimentação e atividade física), relacionando-os ao tema Síndrome Metabólica. 
Além dessas atividades, há fichas de perguntas e desafios que testam os conhecimentos bioquímicos dos jogadores, também relacionados à Síndrome.

O sistema de pontuação baseia-se em 3 pilares: Glicemia, Circunferência Abdominal e HDL (High Density Lipoprotein). As atividades do tabuleiro provocam o aumento ou diminuição de cada um desses fatores e ao final do jogo, com a chegada dos jogadores no HUAP, é possível calcular os valores finais dos parâmetros mencionados anteriormente para cada jogador e fornecer um diagnóstico proporcional à sua pontuação no jogo.

\section{Apresentação do material}

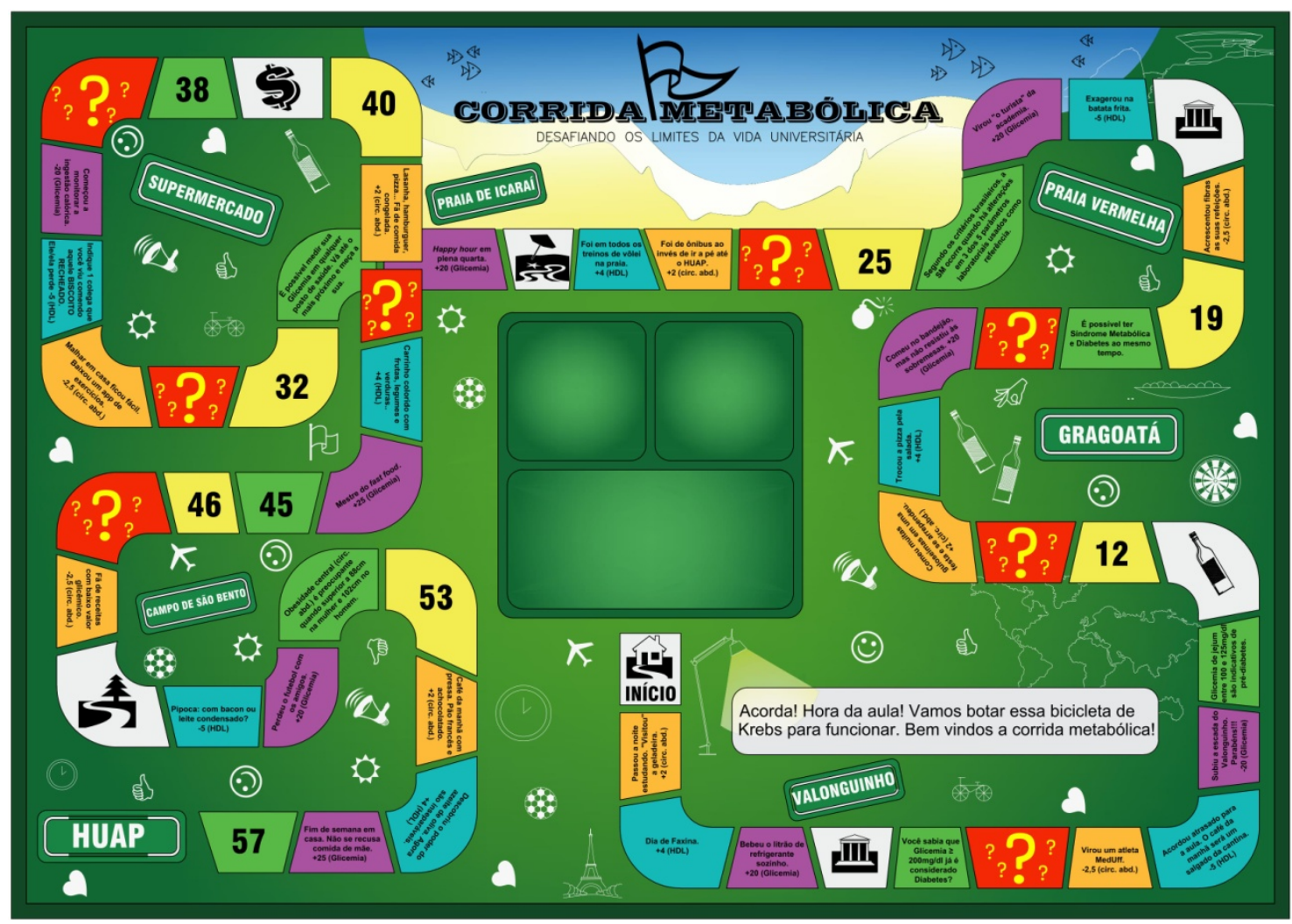

Figura 1. Jogo de Tabuleiro: Corrida Metabólica. 
Quadro 1. Diagnósticos

\begin{tabular}{|c|c|c|}
\hline Diagnóstico & Informações laboratoriais & \\
\hline Saudável & $\begin{array}{l}\text { Glicemia: }<100 \mathrm{mg} / \mathrm{dL} \\
\text { Circunferência abdominal: } \\
\text { Homens: }<102 \mathrm{~cm} \\
\text { Mulheres: }<88 \mathrm{~cm} \\
\mathrm{HDL}: \geq 60 \mathrm{mg} / \mathrm{dL}=\mathrm{HDL} \text { alto (baixo } \\
\text { risco) }\end{array}$ & $\begin{array}{l}\text { Se alimentou de modo equilibrado, } \\
\text { praticou exercícios físicos e utilizou } \\
\text { os conhecimentos bioquímicos a seu } \\
\text { favor. }\end{array}$ \\
\hline Pré-diabetes & Glicemia: $\geq 100 \mathrm{mg} / \mathrm{dL}$ e $<126 \mathrm{mg} / \mathrm{dL}$ & $\begin{array}{l}\text { Glicemia de jejum alterada, tolerância } \\
\text { diminuida à glicose. }\end{array}$ \\
\hline Diabetes Mellitus & Glicemia: $\geq 126 \mathrm{mg} / \mathrm{dL}$ & $\begin{array}{l}\text { Glicemia de jejum: } \geq 126 \mathrm{mg} / \mathrm{dL} ; 2 \mathrm{~h} \\
\text { após } 75 \mathrm{~g} \text { de glicose: } \geq 200 \mathrm{mg} / \mathrm{dL} ; \\
\text { Casual: } \geq 200 \mathrm{mg} / \mathrm{dL} \text { (com sintomas } \\
\text { clássicos) }\end{array}$ \\
\hline HDL baixo & $\mathrm{HDH}:<60 \mathrm{mg} / \mathrm{dL}$ & $\begin{array}{l}\text { Maior risco de desenvolver doenças } \\
\text { cardiovasculares. }\end{array}$ \\
\hline Obesidade abdominal & $\begin{array}{l}\text { Circunferência abdominal: } \\
\text { Homens: }>102 \mathrm{~cm} \\
\text { Mulheres: }>88 \mathrm{~cm}\end{array}$ & $\begin{array}{l}\text { Maior risco de desenvolver doenças } \\
\text { cardiovasculares. }\end{array}$ \\
\hline Sindrome Metabólica & $\begin{array}{l}\text { Glicemia: } \geq 100 \mathrm{mg} / \mathrm{dL} \\
\text { Circunferência Abdominal: } \\
\text { Homens: }>102 \mathrm{~cm} \\
\text { Mulheres: }>88 \mathrm{~cm} \\
\text { HDL } \\
\text { Homens: }<40 \mathrm{mg} / \mathrm{dL} \\
\text { Mulheres: }<50 \mathrm{mg} / \mathrm{dL}\end{array}$ & $\begin{array}{l}\text { Alimentação inadequada e } \\
\text { sedentarismo. }\end{array}$ \\
\hline Síndrome Metabólica + Diabetes & $\begin{array}{l}\text { Glicemia: } \geq 126 \mathrm{mg} / \mathrm{dL} \\
\text { Circunferência Abdominal: } \\
\text { Homens: }>102 \mathrm{~cm} \\
\text { Mulheres: }>88 \mathrm{~cm} \\
\text { HDL } \\
\text { Homens: }<40 \mathrm{mg} / \mathrm{dL} \\
\text { Mulheres: }<50 \mathrm{mg} / \mathrm{dL}\end{array}$ & $\begin{array}{l}\text { Alimentação inadequada e } \\
\text { sedentarismo mais glicemia de jejum } \\
\geq 126 \mathrm{mg} / \mathrm{dL}\end{array}$ \\
\hline
\end{tabular}

\section{Potencial Educacional}

O jogo, com linguagem simples e formato interativo, permite ao estudante revisar e consolidar conhecimentos construídos nas aulas de bioquímica metabólica, com ênfase no tema Síndrome Metabólica.

Tais objetivos são alcançados através dos comandos presentes no tabuleiro e nas fichas de perguntas que promovem alterações nos valores de Glicemia, Circunferência Abdominal e HDL (High Density Lipoprotein), dependendo das atividades realizadas pelos estudantes no jogo.

A inserção dos conteúdos trabalhados em sala de aula em vivência diária, como apresenta o jogo, promove também uma reflexão no estudante acerca do seu estilo de vida.Vale ressaltar que, a realização desta atividade em grupo estimula a socialização e cooperatividade entre os alunos. 


\section{Diferencial com os materiais/ atividade pré-existentes}

O tabuleiro do jogo foi organizado de forma a mimetizar o ambiente e a rotina vivenciada pelos estudantes da UFF, que percorrem durante o jogo um mapa simplificado da cidade de Niterói, no qual são destacados pontos como o Campus Valonguinho, o Hospital Universitário Antônio Pedro (HUAP) e a Praia de Icaraí. Além desses aspectos familiares, os comandos presentes no tabuleiro foram planejados visando uma identificação, por parte dos alunos, quanto a seus hábitos de vida, alimentação e atividade física, relacionando-os ao tema Síndrome Metabólica. Outro diferencial dessa atividade é sua função conscientizadora, uma vez que ao longo do jogo é possível trabalhar noções de prevenção primária e secundária da Síndrome Metabólica e Diabetes Mellitus.

\section{Procedimentos de uso ou aplicação}

A turma é dividida em grupos e em cada um deles um dos alunos desempenha a função de Médico da Família, ficando encarregado de entregar as fichas, ler as atividades e fornecer odiagnóstico quando solicitado no comando do tabuleiro. Cada grupo recebe 1 tabuleiro com pinos, um dado com números de 1 a 6 , fichas de perguntas (64), fichas desafio (48), 1 ficha de diagnósticos e 1 bloco para anotações do médico. O sistema de pontuação baseia-se em 3 critérios dos 5 utilizados para o diagnóstico da Síndrome Metabólica: Glicemia, Circunferência Abdominal e HDL. Cada jogador inicia a partida com: Glicemia: $100 \mathrm{mg} / \mathrm{dL}$, Circunferência abdominal: homem $-84 \mathrm{~cm}$ e mulher $-74 \mathrm{~cm}$ e HDL: homem - $60 \mathrm{mg} / \mathrm{dL}$ e mulher $-50 \mathrm{mg} / \mathrm{dL}$. No final do jogo, é possível calcular as taxas de cada jogador e fornecer um diagnóstico proporcional. $\mathrm{O}$ vencedor é o jogador mais saudável que primeiro chegar ao HUAP.

\subsection{Materiais/ procedimentos}

\begin{tabular}{|c|l|c|}
\hline Material & \multicolumn{1}{|c|}{ Descrição } & Tipo do arquivo \\
\hline $\begin{array}{c}\text { Tabuleiro do } \\
\text { Jogo }\end{array}$ & $\begin{array}{l}\text { Material plastificado no tamanho A2. As casas coloridas em laranja } \\
\text { contém atividades que provocam alterações na circunferência abdominal, } \\
\text { as roxas alteram a glicemia e as de cor azul alteram HDL. As casas } \\
\text { vermelhas com interrogações indicam que o jogador deve pegar uma } \\
\text { ficha de pergunta. As casas brancas que marcam as localidades do mapa } \\
\text { indicam que o jogador deve pegar uma ficha de desafio. As casas verdes } \\
\text { contém informações relevantes sobre a Síndrome Metabólica. }\end{array}$ & Tabuleiro \\
\hline $\begin{array}{c}\text { Regras do } \\
\text { Jogo }\end{array}$ & $\begin{array}{l}\text { Material que acompanha o tabuleiro e contém as regras do jogo, } \\
\text { informando como deve ser organizado, quais as funções de cada jogador } \\
\text { e esclarece possíveis dúvidas. }\end{array}$ & Texto \\
\hline $\begin{array}{c}\text { Tabela de } \\
\text { Diagnósticos }\end{array}$ & \begin{tabular}{l} 
Tabela contendo valores de referência \\
\hline
\end{tabular} & Tabela \\
\hline
\end{tabular}




\section{Considerações finais}

O jogo foi aplicado a 50 alunos do curso de medicina da UFF e apenas $10 \%$ classificou o assunto abordado como difícil. Os alunos avaliaram o jogo como um importante suporte educacional e $85 \%$ mencionaram que foi possível consolidar os conteúdos discutidos em sala de aula com a aplicação do jogo. A dinâmica e a percepção sobre o próprio desempenho ao jogar, também foram muito bem avaliados pelos alunos (100\%). Além disso, 95\% apontaram que iriam jogar novamente e 98\% disseram que pensam que os jogos são capazes de otimizar o aprendizado. Portanto, foi possível com a aplicação de - Uma Corrida Metabólica - não só aproximar os fenômenos bioquímicos ao cotidiano dos alunos, mas também solidificar os conceitos teóricos de forma dinâmica e divertida.

\section{Referências}

[1] Stefanelli MC, Cadete MMM, Aranha MI. Proposta de ação educativa na prevenção da AIDS: jogo educativo. Texto Contexto Enferm, setembro-dezembro. 1998; 7(3):158-73.

[2] Shall VT, Monteiro S, Rebello S, Torres M. Evaluation of the ZIG-ZAIDS game: an entertaining educational tool for HIV/Aids prevention. Cad Saúde Pública. 1999; 15 (Supl 2):107-19.

[3] Fonseca LMM, Scochi CGS, Mello DF. Educação em saúde de puérperas em alojamento conjunto neonatal: aquisição de conhecimento mediado pelo uso de jogo educativo. Rev. Latino Am. Enfermagem março-abril.2002;10(2):166-71.

[4] Torres HC, Hortale VA, Schall VA. A experiência de jogos em grupos operativos na educação em saúde para diabéticos. Cad Saúde Pública, julho-agosto. 2003; 19(4):1039-47.

[5] Monteiro SS, Vargas EP, Rebello SM. Educação, prevenção e drogas: resultados e desdobramentos da avaliação de um jogo educativo. Edu Soc agosto. 2003; 24(83):659-78.

[6] Yonekura T, Soares CB. O jogo educativo como estratégia de sensibilização para coleta de dados com adolescentes. Rev. Latino-Am. Enfermagem.2010;18(5).

[7]Sociedade Brasileira de Endocrinologia e Metabologia. 2016. Available from:https://www.endocrino.org.br/sindrome-metabolica/ 